\title{
GERMINAÇÃO E SANIDADE DE SEMENTES DE CUPUAÇU (Theobroma grandiflorum) TRATADAS COM FUNGINCIDAS
}

\author{
A. G. SANCHES*, E. G. S. MOREIRA, J. M. COSTA, M. B. SILVA e C. A. M. CORDEIRO \\ Universidade Federal do Pará, Campus Altamira-Pará \\ alexsanches.eng@gmail.com*
}

Artigo submetido em dezembro/2015 e aceito em dezembro/2015

DOI: $10.15628 /$ holos.2015.3825

\section{RESUMO}

A qualidade da semente é fator de extrema importância para que se obtenha uma muda sadia e consequentemente uma boa produtividade, e o armazenamento é prática fundamental para o controle da qualidade fisiológica da semente. Assim, o presente trabalho tem por objetivo avaliar o potencial germinativo e a sanidade de sementes de cupuaçu tratadas com diferentes fungicidas por um período de seis meses. As sementes avaliadas foram da variedade redondo, estas foram submetidas a seis diferentes tratamentos com fungicidas e armazenadas a temperatura de $5^{\circ} \mathrm{C}$ por seis meses. A cada dois meses avaliaram-se a porcentagem de germinação, IVE, teste de frio, envelhecimento acelerado, teor de água, condutividade elétrica e sanidade das sementes. Dentre as características avaliadas não houve interação somente para teor de água e condutividade elétrica. Observou-se efeito positivo dos tratamentos com fungicidas no controle dos fungos sem comprometer a germinação quando comparados a testemunha. O fungicida Captan apresentou os melhores resultados para todas as características avaliadas. Verificou-se ainda que o potencial germinativo é comprometido após dois meses de armazenamento independente do tratamento utilizado.

PALAVRAS-CHAVE: Compostos químicos, fungos, vigor.

\section{CUPUAÇU GERMINATION AND SEED HEALTH (Theobroma grandiflorum) TREATED WITH FUNGINCIDAS}

\begin{abstract}
Seed quality is extremely important factor in order to obtain a sound change and consequently a high productivity, and storage is essential to control the circulation of physiological seed quality. Thus, this study aims to evaluate the germination potential and the sanity of cupuaçu seeds treated with fungicides for a period of six months. The evaluated were round selection, these were subjected to six different fungicide treatments and stored at $5^{\circ} \mathrm{C}$ for six months. Every two months evaluated the percentage of germination, EVI, cold test,
\end{abstract}

accelerated aging, water content, electrical conductivity and seed health. Among the characteristics evaluated there was no interaction only for water content and electrical conductivity. It was observed positive effect of treatments with fungicides to control the fungus without compromising germination when compared to control. The Captan fungicide showed the best results for all traits. It was also found that the germination potential is compromised after two months of storage regardless of treatment used.

KEYWORDS: Chemical compounds, fungi, force. 


\section{INTRODUÇÃO}

A qualidade da semente é fator de extrema importância para que se obtenha uma muda sadia e consequentemente uma boa produtividade, e o armazenamento é prática fundamental para o controle da qualidade fisiológica da semente, sendo um método por meio do qual, pode-se preservar a viabilidade das sementes e manter o vigor em nível razoável no período compreendido entre o plantio e a colheita (AZEVEDO et al., 2003). O nível de deterioração em sementes armazenadas depende das condições do lote por ocasião do início da armazenagem e do controle dos fatores ambientais durante essa fase (FREITAS et al., 2000). Outro fator importante a ser considerado é a incidência de fungos no armazenamento (LACERDA et al., 2003), constituindo-se, juntamente com os insetos, as principais causas de deterioração e perdas (SANTOS, 2006).

O tratamento de sementes com fungicidas é uma prática que tem sido recomendada, visando não só a preservação da qualidade das sementes, mas também melhorar o desempenho germinativo destas sob condições adversas (GOULART et al., 2000; PEREIRA et al., 2007).

As sementes de cupuaçu são facilmente afetadas por patógenos devido à presença de mucilagem sobre a mesma sendo este um atrativo para a proliferação de microorganismos associados a alta umidade nos locais de produção (CRUZ et al., 2006). Além do que as sementes de cupuaçu são do tipo recalcitrantes, isto é, apresentam tamanho relativamente grande e são caracterizadas por não sofrerem dessecação natural na planta-mãe ao longo do processo de maturação, sendo dispersas com elevados teores de água que, se reduzidos a um nível considerado crítico, levarão à rápida perda da viabilidade e até a morte, necessitando, portanto, de técnicas que prolonguem sua qualidade fisiológica

A literatura é carente de informações para a adequada conservação de sementes recalcitrantes, especialmente o cupuaçu. Com base nessas considerações, o presente trabalho teve como objetivo avaliar o efeito do tratamento com fungicidas durante o armazenamento de sementes de cupuaçu.

\section{METODOLOGIA}

Os frutos de cupuaçuzeiro (Theobroma grandiflorum) da variedade "Redondo" foram adquiridos no campo experimental da Embrapa Amazônia Oriental localizado no município de Altamira-PA e transportados em caixas de papelão até o laboratório de Multidisciplinar da Universidade Federal do Pará, Campus Altamira onde foram quebrados e despolpados.

Inicialmente um lote representativo de 100 sementes foram caracterizadas quanto a porcentagem de germinação, estas foram colocadas sobre papel "Germitest" e mantidas em germinador a $25^{\circ} \mathrm{C}$ obtendo um percentual de $96 \%$. Quanto a sanidade houve a incidência de $21 \%$ de Aspergillus sp, $15 \%$ de Fusarium spp e $12 \%$ de Colletotrichum gossypii, estes foram determinados avaliando o percentual de sementes afetadas tendo como base o total de $100 \%$ de sementes do lote avaliado. Para a aplicação dos tratamentos as sementes foram homogeneizadas em relação ao tamanho e o peso e submetidas aos seguintes tratamentos com os fungicidas: $\mathrm{T} 1=$ Testemunha (sem tratamento antifúngico); T2= Captan; T3= Tebuconazol, T4=Carbendazin, T5=Thiran, T6= Abamectina e T7= Fipronil. 
O volume de cada fungicida (200g) foi diluído em 1 litro de água para cada $100 \mathrm{~kg}$ de sementes conforme recomendação do fabricante. As sementes foram acondicionadas em bandejas plásticas e imersas nos produtos até a completa distribuição do produto. $O$ tratamento testemunha foi mantido em água destilada por igual período. Após a aplicação, as sementes de cada tratamento foram mantidas em local seco e arejado até estarem secas, posteriormente estas foram acondicionadas em embalagens permeáveis (sacos de papel Kraft) devidamente identificadas e armazenadas em B.O.D a $5^{\circ} \mathrm{C}$ por um período de seis meses sendo avaliadas a cada dois meses quanto as seguintes características:

a) A porcentagem de germinação foi realizada com quatro amostras compostas por 50 sementes cada, estas foram semeadas em papel "Germitest", umedecido com água destilada, na proporção de 2,5 vezes o peso do papel seco e foram mantidas em germinador a $25^{\circ} \mathrm{C}$. As avaliações foram realizadas segundo as Regras para Análise de Sementes (BRASIL, 2005). A contagem de plântulas normais foi realizada após o terceiro dia de semeadura fazendo a contagem de plântulas normais germinadas.

b) $O$ índice de velocidade de emergência (IVE), foi determinado utilizando a fórmula proposta por Maguirre (1962), onde foi registrado a partir do terceiro dia o número de sementes germinadas até o $12 \%$ dia após a semeadura.

c) O teste de frio foi realizado em câmara fria onde quatro amostras composta por 50 sementes cada foram acondicionadas em potes de polipropileno e mantidas $a 10^{\circ} \mathrm{C}$ por dez dias sendo posteriormente plantadas em sacos de polietileno contendo areia + substrato comercial Plantmax de onde procedeu-se a contagem de sementes germinadas.

d) O teste de envelhecimento acelerado foi determinado conforme metodologia descrita por Marcos Filho (1999), utilizando-se para cada lote 400 sementes distribuídas em camada uniforme sobre tela metálica acoplada a caixas plásticas transparentes, contendo $40 \mathrm{~cm} 3$ de água destilada ao fundo. Os recipientes foram mantidos sob temperatura de 42 ㅇ durante 48 horas, conforme Brasil (2009).

e) O teor de água das sementes foi determinado pelo método da estufa a 105드 por 24 horas, sendo utilizadas duas repetições de $500 \mathrm{~g}$ sementes, por tratamento de acordo com as Regras para Análise de Sementes - descrita por Brasil (2005).

f) A condutividade elétrica foi realizada utilizando quatro repetições de 50 sementes para cada tratamento, pesadas com precisão de $0,01 \mathrm{~g}$ e colocadas para embeber em copos plásticos contendo 50 e $75 \mathrm{~mL}$ de água deionizada, a 25으, durante 12,18 e 24 horas. Após cada período de embebição, procedeu-se à leitura da condutividade elétrica do exsudato, em um condutivímetro DIGIMED CD-20, sendo os resultados expressos em $\mu \mathrm{S} \mathrm{cm}^{-1} \mathrm{~g}^{-1}$.

g) O teste de sanidade foi realizado por meio de incubação das sementes em placas de Petri contendo três folhas de papel de filtro umedecido com água + 2,4-D (2,4diclorofenoxiacetato de sódio) a 0,02\%. Foram utilizadas 25 sementes de cada tratamento por placa, num total de oito amostras. As placas foram mantidas em incubadora a 20 으 e fotoperíodo de 12 horas, por sete dias, e posteriormente avaliadas quanto à presença de patógenos sendo os resultados expressos em porcentagem. 
O delineamento experimental adotado foi o inteiramente casualizado em esquema fatorial $7 \times 4$ sendo: (sete tratamentos químicos e quatro tempos de avaliação: 0, 2, 4 e 6 meses) com cinco repetições por tratamento. Os dados foram submetidos à análise de variância utilizando o programa estatístico Bioestat versão 4.2. As médias entre os tratamentos foram comparadas pelo teste de Tukey, ao nível de $5 \%$ de probabilidade.

\section{RESULTADOS E DISCUSSÕES}

De acordo com a tabela 1observa-se que independente do tratamento químico utilizado houve redução do número de plântulas germinadas com o tempo de armazenamento e que as sementes mantidas sem tratamento (testemunha) apresentaram os menores percentuais em todos os tempos de armazenamento.

No tempo zero de armazenamento, os dados não mostraram diferenças significativa entre os tratamentos e a testemunha. A avaliação realizada após 2 meses de armazenamento mostrou valores estatisticamente iguais para os tratamentos Tebuconazol, Carbendazin e a testemunha que diferiram significativamente dos demais tratamentos (Tabela 1).

Com quatro meses de armazenamento as sementes foram novamente avaliadas e os tratamentos compostos por Tebuconazol, Carbendazin, Abamectina e a testemunha apresentaram valores semelhantes não diferindo entre si. Para esse mesmo tempo de avaliação as sementes tratadas com Captan, Thiran e Fipronil apresentaram os melhores percentuais de germinação com valores acima de $70 \%$ diferindo significativamente dos demais. No último período de avaliação (6 meses) verifica-se que apenas as sementes tratadas com Captan e Fipronil obtiveram percentual de germinação superior a $60 \%$ diferindo significativamente dos tratamentos com Tebuconazol, Carbendazin, Thiran e Abamectina cujas médias foram de 45,84, 49,81, 51,17 e 52,86 respectivamente. As sementes armazenadas sem tratamento (testemunha) apresentaram 0 menor percentual com média de apenas $32,5 \%$ de germinação ao fim de seis meses de armazenamento (Tabela 1).

Ainda de acordo com a tabela 1, observa-se que mesmo com a redução da germinação com o passar do tempo de armazenamento, os tratamentos com fungicidas apresentaram resultados superiores estatisticamente quando comparadas as sementes sem tratamento químico (testemunha) cuja redução foi mais acentuada à medida que aumentou o período de armazenamento.

Esse fato corrobora com os estudos de Freitas et al. (2006) que ao avaliarem o desempenho fisiológico de sementes de algodão tratada com fungicidas ao longo do tempo de armazenamento observaram melhor desempenho germinativo quando tratadas quimicamente.

Para germinação, os valores obtidos ficaram todos acima de $50 \%$ após seis meses de armazenamento quando comparados a testemunha revelando que os fungicidas não comprometeram a qualidade fisiológica das sementes. Os autores Gagliardi et al. (2009) e Pinto et al. (2011) também constataram que os fungicidas não influenciaram no potencial germinativo das sementes de soja. 
Tabela 1. Média para a variável porcentagem de germinação de sementes de cupuaçu da variedade "Redondo" tratada com fungicidas ao longo do tempo de armazenamento.

\begin{tabular}{ccccc}
\hline Tratamentos & \multicolumn{4}{c}{ Tempos de armazenamento } \\
\cline { 2 - 5 } & 0 & 2 & 4 & 6 \\
Testemunha & $92,55 \mathrm{a}$ & $78,46 \mathrm{~b}$ & $52,86 \mathrm{~b}$ & $32,51 \mathrm{c}$ \\
Captan & $93,18 \mathrm{a}$ & $87,67 \mathrm{a}$ & $76,64 \mathrm{a}$ & $68,43 \mathrm{a}$ \\
Tebuconazol & $91,84 \mathrm{a}$ & $78,95 \mathrm{~b}$ & $56,19 \mathrm{~b}$ & $45,84 \mathrm{~b}$ \\
Carbendazin & $93,25 \mathrm{a}$ & $79,18 \mathrm{~b}$ & $55,07 \mathrm{~b}$ & $49,81 \mathrm{~b}$ \\
Thiran & $94,57 \mathrm{a}$ & $86,19 \mathrm{a}$ & $77,57 \mathrm{a}$ & $51,17 \mathrm{~b}$ \\
Abamectina & $91,84 \mathrm{a}$ & $85,59 \mathrm{a}$ & $56,87 \mathrm{~b}$ & $52,16 \mathrm{~b}$ \\
Fipronil & $92,58 \mathrm{a}$ & $88,09 \mathrm{a}$ & $76,53 \mathrm{a}$ & $68,39 \mathrm{a}$ \\
\hline
\end{tabular}

Médias seguidas pela mesma letra na coluna não diferem entre si. Foi aplicado o teste de tukey a $5 \%$ de probabilidade.

No que se refere ao índice de velocidade de emergência, observa-se decréscimo nos percentuais à medida que houve aumento no período de armazenamento das sementes independente do tratamento aplicado (Tabela 2).

No tempo zero armazenamento os valores não mostraram significância entre os tratamentos apresentando percentuais superiores a $20 \%$. Aos dois meses de análise o vigor das sementes reduziu significativamente com maior diferença para os tratamentos testemunha, Tebuconazol e Carbendazin que apresentaram médias de apenas 14,85 e 14,91 e 15,82\% respectivamente enquanto que para as sementes tratadas com Captan, Thiran, Abamectina e Fipronil o percentual médio para o mesmo período variou entre 18 e 19\% (Tabela 2).

Quanto ao índice de velocidade de germinação com quatro meses de armazenamento apenas os tratamentos compostos por Captan, Thiran e Fipronil foram estatisticamente superiores apresentando médias de 12,61, 12,54 e 12,57 respectivamente. As sementes tratadas com Tebuconazol e Carbendazin apresentaram médias de 9,74 e 9,82 respectivamente sendo estes inferiores ao verificado pela testemunha cuja média foi de $10,55 \%$. As sementes tratadas com Abamectina apresentaram percentual de 10,02, contudo não diferiu dos demais tratamentos.

Tabela 2. Média para a variável índice de velocidade de germinação a partir do teste de germinação de sementes de cupuaçu da variedade "Redondo" tratada com fungicidas ao longo do tempo de armazenamento.

\begin{tabular}{ccccc}
\hline Tratamentos & \multicolumn{4}{c}{ Tempos de armazenamento } \\
\cline { 3 - 5 } & 0 & 2 & 4 & 6 \\
Testemunha & $22,54 \mathrm{a}$ & $14,85 \mathrm{~b}$ & $10,55 \mathrm{~b}$ & $5,54 \mathrm{a}$ \\
Captan & $22,89 \mathrm{a}$ & $18,74 \mathrm{a}$ & $12,61 \mathrm{a}$ & $9,54 \mathrm{a}$ \\
Tebuconazol & $22,57 \mathrm{a}$ & $14,91 \mathrm{~b}$ & $9,74 \mathrm{~b}$ & $5,96 \mathrm{a}$ \\
Carbendazin & $23,82 \mathrm{a}$ & $15,82 \mathrm{~b}$ & $9,82 \mathrm{~b}$ & $6,44 \mathrm{a}$ \\
Thiran & $21,76 \mathrm{a}$ & $18,89 \mathrm{a}$ & $12,54 \mathrm{a}$ & $9,39 \mathrm{a}$ \\
Abamectina & $22,04 \mathrm{a}$ & $19,15 \mathrm{a}$ & $10,02 \mathrm{~b}$ & $7,35 \mathrm{a}$ \\
Fipronil & $22,13 \mathrm{a}$ & $19,08 \mathrm{a}$ & $12,57 \mathrm{a}$ & $7,71 \mathrm{a}$ \\
\hline
\end{tabular}

Médias seguidas pela mesma letra na coluna não diferem entre si. Foi aplicado o teste de tukey a $5 \%$ de probabilidade.

Aos seis meses de armazenamento o índice de velocidade de germinação não diferiu entre os tratamentos aplicados e em relação a testemunha, todavia as sementes mantidas com Captan e Thiran apresentaram os maiores valores (Tabela 2). A redução do vigor das sementes de cupuaçu com o tempo de armazenamento pode estar relacionada a associação de microrganismos saprofíticos e ou fungos de armazenamento que se aproveitam das reservas das sementes para se manter. 
No trabalho de Chitarra et al. (2008) o índice de velocidade de germinação das sementes de algodão com fungicidas foi significativamente superior quando comparados à testemunha independente do tempo de armazenamento.

A porcentagem de germinação das sementes a partir do teste de frio apresentou percentuais semelhantes aos observados pelo teste de germinação realizado normalmente, observando redução nos valores entre o tempo de zero a seis meses (Tabela 3). As sementes mantidas sem tratamento apresentaram os menores percentuais durante todo o período de armazenamento sendo mais significativo esse percentual após dois meses de armazenamento quando registrou média de apenas $45,58 \%$ em relação aos tratamentos químicos que para 0 mesmo período apresentaram média variando entre 65 e 74\% (Tabela 3).

Após quatro meses de armazenamento em câmara fria, verifica-se uma estabilidade no percentual de germinação em todos os tratamentos químicos com valores médios oscilando entre 50 e $55 \%$, as sementes mantidas sem tratamento (testemunha) apresentaram percentual de apenas 33,77\% diferindo significativamente dos demais (Tabela 3). Aos 6 meses de armazenamento (Tabela 3), quando as sementes foram tratadas com Tebuconazol, Carbendazin, Abamectina e Fipronil, a porcentagem de germinação pelo teste de frio foram iguais a da testemunha e menores que de todos os outros tratamentos. Pereira et al. (2007) também verificaram melhor desempenho germinativo após teste de frio em sementes de soja tratadas com fungicidas quando comparadas a testemunha.

Tabela 3. Média para a variável porcentagem de germinação pelo teste de frio em sementes de cupuaçu da variedade "Redondo" tratada com fungicidas ao longo do tempo de armazenamento.

\begin{tabular}{|c|c|c|c|c|}
\hline \multirow{2}{*}{ Tratamentos } & \multicolumn{4}{|c|}{ Tempos de armazenamento } \\
\hline & 0 & 2 & 4 & 6 \\
\hline Testemunha & 91,47 a & $45,58 \mathrm{~b}$ & $33,77 b$ & $27,93 \mathrm{~b}$ \\
\hline Captan & 90,19 a & $73,45 \mathrm{a}$ & $54,89 a$ & 43,19 a \\
\hline Tebuconazol & $90,56 \mathrm{a}$ & $66,54 a$ & $52,47 a$ & $29,89 b$ \\
\hline Carbendazin & 93,18 a & 67,12 a & $50,89 a$ & $28,31 \mathrm{~b}$ \\
\hline Thiran & 91,19 a & $71,89 \mathrm{a}$ & $53,54 a$ & 40,87 a \\
\hline Abamectina & $90,07 \mathrm{a}$ & $68,45 \mathrm{a}$ & $51,08 \mathrm{a}$ & $28,87 \mathrm{~b}$ \\
\hline Fipronil & 89,67 a & $73,77 \mathrm{a}$ & $54,27 a$ & $28,53 \mathrm{~b}$ \\
\hline
\end{tabular}

Médias seguidas pela mesma letra na coluna não diferem entre si. Foi aplicado o teste de tukey a $5 \%$ de probabilidade.

No que concerne a germinação das sementes após o teste de envelhecimento acelerado, observa-se variação nos valores ao longo do armazenamento em todos os tratamentos (Tabela 4). As sementes tratadas com Captan, Thiran e Fipronil apresentaram maior redução a partir do quarto mês de armazenamento enquanto que os tratamentos formados por Tebuconazol, Carbendazin e testemunha a redução foi progressiva já no segundo mês de análise (Tabela 4).

No tempo zero de armazenamento não foi observado interação significativa entre os tratamentos formados por Captan, Thiran e Fipronil em relação a testemunha que apresentaram resultados semelhantes, todavia as sementes tratadas com Tebuconazol, Carbendazin e Abamectina apresentaram baixo nível de germinação podendo inferir que essas substâncias químicas podem ter causado efeito tóxico as sementes, nas condições do teste inibindo sua germinação.

Após o armazenamento por quatro meses, o percentual de germinação das sementes tratadas com Tebuconazol, Carbendazin e Abamectina foram estatisticamente iguais ao da 
testemunha e inferiores ao dos demais produtos. As sementes tratadas por Captan, Thiran e Fipronil não diferiram entre si, todavia mostraram resultados superiores aos demais tratamentos evidenciando pouca queda no percentual germinativo das sementes (Tabela 4).

Quando as sementes foram armazenadas por 6 meses os tratamentos apresentaram percentual de germinação abaixo de $30 \%$ em todos os tratamentos (Tabela 4). As sementes tratadas com Captan e Fipronil apresentaram percentuais de 28,75 e 28,93\% diferindo estatisticamente dos tratamentos Tebuconazol, Carbendazin, Thiran, Abamectina e testemunha que apresentaram médias de 20,14, 20,04, 19,87, 19,65 e 16,93 respectivamente (Tabela 4).

Tabela 5. Média para porcentagem de germinação pelo teste de envelhecimento acelerado em sementes de cupuaçu da variedade "Redondo" tratada com fungicidas ao longo do tempo de armazenamento.

\begin{tabular}{cccccc}
\hline Tratamentos & \multicolumn{4}{c}{ Tempos de armazenamento } \\
\cline { 3 - 6 } & 0 & 2 & 4 & 6 \\
Testemunha & $88,54 \mathrm{a}$ & $53,57 \mathrm{~b}$ & $31,87 \mathrm{~b}$ & $16,93 \mathrm{~b}$ \\
Captan & $89,51 \mathrm{a}$ & $64,89 \mathrm{a}$ & $54,89 \mathrm{a}$ & $28,75 \mathrm{a}$ \\
Tebuconazol & $74,87 \mathrm{~b}$ & $54,89 \mathrm{~b}$ & $35,42 \mathrm{~b}$ & $20,14 \mathrm{~b}$ \\
Carbendazin & $75,98 \mathrm{~b}$ & $57,55 \mathrm{~b}$ & $37,54 \mathrm{~b}$ & $20,04 \mathrm{~b}$ \\
Thiran & $86,58 \mathrm{a}$ & $66,17 \mathrm{a}$ & $55,19 \mathrm{a}$ & $19,87 \mathrm{~b}$ \\
Abamectina & $76,32 \mathrm{~b}$ & $54,18 \mathrm{~b}$ & $33,21 \mathrm{~b}$ & $19,65 \mathrm{~b}$ \\
Fipronil & $87,36 \mathrm{a}$ & $63,76 \mathrm{a}$ & $53,85 \mathrm{a}$ & $28,93 \mathrm{a}$ \\
\hline
\end{tabular}

Médias seguidas pela mesma letra na coluna não diferem entre si. Foi aplicado o teste de tukey a 5\% de probabilidade.

Não houve diferença significativa para as variáveis teor de água e condutividade elétrica tabelas 5 e 6 respectivamente, durante todo o período de armazenamento.

Ao longo dos seis meses de armazenamento das sementes de cupuaçu houve aumento da perda de água em todos os tratamentos (Tabela 5) e com isso houve maior lixiviação dos exsudatos que compõem as sementes (Tabela 6) indicando, portanto, maior desorganização dos sistemas membranais das células presentes nas sementes evidenciando, assim, menor potencial fisiológico com o tempo de armazenamento.

As sementes mantidas sem tratamento apresentaram as maiores perdas de água e consequentemente evidenciaram menor condutividade elétrica, no entanto não diferiu das sementes tratadas com fungicidas (Tabela 5).

Tabela 5: Valores médios sobre a variável teor de água das sementes de cupuaçu, variedade "Redondo" tratado com fungicidas ao longo do tempo de armazenamento.

\begin{tabular}{ccccc}
\hline Tratamentos & \multicolumn{4}{c}{ Tempos de armazenamento } \\
\cline { 3 - 5 } & 0 & 2 & 4 & 6 \\
Testemunha & $63,4 \mathrm{a}$ & $56,34 \mathrm{a}$ & $49,19 \mathrm{a}$ & $44,17 \mathrm{a}$ \\
Captan & $62,8 \mathrm{a}$ & $59,25 \mathrm{a}$ & $50,38 \mathrm{a}$ & $44,29 \mathrm{a}$ \\
Tebuconazol & $63,2 \mathrm{a}$ & $56,83 \mathrm{a}$ & $49,37 \mathrm{a}$ & $44,21 \mathrm{a}$ \\
Carbendazin & $62,8 \mathrm{a}$ & $61,57 \mathrm{a}$ & $49,56 \mathrm{a}$ & $44,05 \mathrm{a}$ \\
Thiran & $61,7 \mathrm{a}$ & $58,34 \mathrm{a}$ & $50,17 \mathrm{a}$ & $44,33 \mathrm{a}$ \\
Abamectina & $64,4 \mathrm{a}$ & $56,89 \mathrm{a}$ & $49,23 \mathrm{a}$ & $44,86 \mathrm{a}$ \\
Fipronil & $63,8 \mathrm{a}$ & $59,27 \mathrm{a}$ & $50,43 \mathrm{a}$ & $44,57 \mathrm{a}$ \\
\hline
\end{tabular}

Médias seguidas pela mesma letra na coluna não diferem entre si. Foi aplicado o teste de tukey a $5 \%$ de probabilidade. 
Tabela 6: Valores médios sobre a variável condutividade elétrica das sementes de cupuaçu, variedade "Redondo" tratado com fungicidas ao longo do tempo de armazenamento.

\begin{tabular}{cccccc}
\hline \multirow{2}{*}{ Tratamentos } & \multicolumn{4}{c}{ Tempos de armazenamento } \\
\cline { 3 - 5 } \cline { 3 - 5 } & 0 & 2 & 4 & 6 \\
Testemunha & $13,49 \mathrm{a}$ & $9,87 \mathrm{a}$ & $7,79 \mathrm{a}$ & $5,17 \mathrm{a}$ \\
Captan & & $13,83 \mathrm{a}$ & $10,21 \mathrm{a}$ & $8,15 \mathrm{a}$ & $5,73 \mathrm{a}$ \\
Tebuconazol & $13,75 \mathrm{a}$ & $10,05 \mathrm{a}$ & $7,91 \mathrm{a}$ & $5,44 \mathrm{a}$ \\
Carbendazin & $13,75 \mathrm{a}$ & $9,89 \mathrm{a}$ & $7,58 \mathrm{a}$ & $5,32 \mathrm{a}$ & $5,23 \mathrm{a}$ \\
Thiran & $13,21 \mathrm{a}$ & $9,96 \mathrm{a}$ & $8,13 \mathrm{a}$ & $5,28 \mathrm{a}$ \\
Abamectina & $13,55 \mathrm{a}$ & $10,18 \mathrm{a}$ & $7,89 \mathrm{a}$ & $5,44 \mathrm{a}$ \\
Fipronil & $13,17 \mathrm{a}$ & $10,22 \mathrm{a}$ & $8,09 \mathrm{a}$ & \\
\hline
\end{tabular}

CV $(\%)=7,17$

Médias seguidas pela mesma letra na coluna não diferem entre si. Foi aplicado o teste de tukey a 5\% de probabilidade.

De acordo com o teste de sanidade Tabela 7 foram identificadas quatro espécies de fungos nos diferentes tratamentos avaliados, sendo eles: Aspergillus sp.; Penincillium sp.; Fusarium spp. (F) e Colletotrichum gossypii. Para as sementes mantidas sem tratamento (testemunha), verificouse que a porcentagem de incidência de fungos após dois meses de armazenamento foi de até 26,8 \% para Aspergillus sp. e 28,54\% para Fusarium spp, enquanto que nos tratamentos químicos não se verificou incidência de patógenos (Tabela 7).

No tempo de quatro meses de armazenamento identificou-se a presença de Penincillium sp. e um aumento no percentual de Aspergillus sp. com médias de 25,4 e 37,5\% respectivamente nas sementes mantidas sem tratamento (testemunha). As sementes tratadas com Tebuconazol, Thiran e Fipronil apresentaram evidências de até $10 \%$ para os fungos Aspergillus sp. e Fusarium sp respectivamente, enquanto que os tratamentos com Captan, Carbendazin e Abamectina houve a constatação de Colletotrichum gossypii em torno de 8,50\% e de Aspergillus sp. com 5,12\% para ambos os tratamentos (Tabela 7).

Após seis meses de armazenamento as sementes tratadas com Tebuconazol, Thiran e Fipronil reduziram a incidência dos fungos Aspergillus sp. e Fusarium sp para até 2,5\%. As sementes tratadas com Captan, Carbendazin e Abamectina mostraram-se isentas de fungos, controlando, portanto, a ocorrência de Colletotrichum gossypii e de Aspergillus sp registrados no quarto mês de armazenamento (Tabela 7). As sementes mantidas sem tratamento apresentaram ocorrência de todos os fungos em até $50 \%$ das amostras com maiores evidências para Aspergillus sp.

De modo geral, os tratamentos foram eficientes no controle da microflora sem comprometer ainda a germinação e a emergência das plântulas, comprovando assim os estudos descritos por Barbosa (2011), Marchi (2010) e Viegas et al. (2005) que avaliaram a qualidade fisiológica de sementes sob diferentes tipos de fungicidas.

Dentre os fungicidas avaliados, destaque para o Captan que apresentou as menores incidências. Este fator positivo apresentado pelo Captan também já foi verificado por Gonçalves et al. (2009) ao avaliarem a qualidade fisiológica de sementes de feijão por um período de seis meses. 
Tabela 7: Incidência de fungos (\%) no armazenamento de sementes de cupuaçu da variedade "Redondo" tratado com fungicidas ao longo do tempo de armazenamento.

\begin{tabular}{|c|c|c|c|c|}
\hline \multirow{2}{*}{ Tratamentos } & \multicolumn{4}{|c|}{2 meses } \\
\hline & Aspergillus sp & Colletotrichum gossypii & Fusarium spp & Penincillium sp. \\
\hline Testemunha & 26,8 & 0,00 & 28,54 & 0,00 \\
\hline Captan & 0,00 & 0,00 & 0,00 & 0,00 \\
\hline Tebuconazol & 0,00 & 0,00 & 0,00 & 0,00 \\
\hline Carbendazin & 0,00 & 0,00 & 0,00 & 0,00 \\
\hline Thiran & 0,00 & 0,00 & 0,00 & 0,00 \\
\hline Abamectina & 0,00 & 0,00 & 0,00 & 0,00 \\
\hline Fipronil & 0,00 & 0,00 & 0,00 & 0,00 \\
\hline \multicolumn{5}{|c|}{4 meses } \\
\hline Testemunha & 37,5 & 0,00 & 0,00 & 25,4 \\
\hline Captan & 4,37 & 5,78 & 0,00 & 0,00 \\
\hline Tebuconazol & 6,44 & 0,00 & 10,00 & 0,00 \\
\hline Carbendazin & 5,12 & 6,89 & 0,00 & 0,00 \\
\hline Thiran & 10,00 & 0,00 & 5,86 & 0,00 \\
\hline Abamectina & 4,93 & 8,50 & 0,00 & 0,00 \\
\hline Fipronil & 6,89 & 0,00 & 8,57 & 0,00 \\
\hline \multicolumn{5}{|c|}{6 meses } \\
\hline Testemunha & 50,0 & 33,90 & 39,57 & 41,9 \\
\hline Captan & 0,00 & 0,00 & 0,00 & 0,00 \\
\hline Tebuconazol & 2,44 & 0,00 & 1,85 & 0,00 \\
\hline Carbendazin & 0,00 & 0,00 & 0,00 & 0,00 \\
\hline Thiran & 2,33 & 0,00 & 2,50 & 0,00 \\
\hline Abamectina & 4,93 & 0,00 & 0,00 & 0,00 \\
\hline Fipronil & 2,50 & 0,00 & 1,68 & 0,00 \\
\hline
\end{tabular}

\section{CONCLUSÕES}

A germinação das sementes de cupuaçu não foi afetada com o uso dos fungicidas, observando ainda manutenção do desempenho fisiológico com o tempo de armazenamento do que aquelas não tratadas.

Os tratamentos utilizados foram eficientes para o controle de fungos de armazenamento.

O fungicida Captan apresentou os melhores resultados tanto para o controle de fungos quanto na germinação das sementes com o tempo de armazenamento.

Independente do tratamento químico, recomenda-se o armazenamento das sementes de cupuaçu por até dois meses uma vez que ultrapassado este período a qualidade fisiológica das sementes fica comprometida.

\section{REFERÊNCIAS BIBLIOGRÁFICAS}

1. AZEVEDO, C. F. Qualidade de sementes e morfoanatomia de plântulas de Foeniculum vulgare 
Mill. oriundas de sistemas de consórcio. Areia, 2009. Dissertação de Mestrado em AgronomiaUniversidade Federal da Paraíba, Areia, 2009.

2. BARBOSA, R. M. Controle químico de patógenos e desempenho fisiológico de sementes de amendoim. Jaboticabal, 2011. Dissertação de Mestrado em Produção Vegetal Faculdade de Ciências Agrárias e Veterinárias-UNESP, Jaboticabal, 2011.

3. BRASIL. Ministério da Agricultura, Pecuária e Abastecimento. Regras para análise de sementes. Brasília: Mapa/ACS, 2009. 399 p.

4. ChitARRA, L. G., GOULART, A. C. P., ZORATO, M. F. Tratamento de sementes de algodoeiro com fungicidas no controle de patógenos causadores de tombamento de plântulas. Revista Brasileira de Sementes, v. 31, n. 1, p.168-176, 2008.

5. COSTA, C. J. Deterioração e Armazenamento de Sementes de Hortaliças Embrapa Clima Temperado. Pelotas: Empresa Brasileira de Pesquisa Agropecuária Embrapa Clima Temperado. 2012. 26p. (Circular Técnica, 355).

6. CRUZ, E. D. Armazenamento de sementes de cupuaçu (Theobroma grandiflorum (Willd. ex Spreng.) K. Schum.), Piracicaba, 2006. Tese de Doutorado - Escola Superior de Agricultura Luiz de Queiroz, Piracicaba, 2007.

7. FREITAS, R. A. et al. Qualidade fisiológica e sanitária de sementes de algodão durante o armazenamento. Revista Brasileira de Sementes, v. 22, n. 2, p.94-101, 2000.

8. GAGLIARDI, B., CARVALHO, T. C., PUPIM, T. L., GOMES JUNIOR, F. G., TIMÓTEO, T. S., KOBORIS, N. M., MORAES, M. L. D., MENTEM, J. O. M. Efeito de fungicidas para controle da ferrugem asiática na qualidade de sementes de soja. Revista Brasileira de Sementes, v. 31, n. 4, p.120125, 2009.

9. GONÇALVES, E. P., URSULINO, E. A., COSTA, N. P. da. Tratamento químico e natural sobre a qualidade fisiológica e sanitária em sementes de feijão (Phaseolus vulgaris I.) armazenadas. Revista de biociências. Taubaté, v. 9, n. 1, p. 23-29, 2004.

10. Goulart, A. C. P., ANDRADE, P. J. M., BORGES, E. P. Controle de patógenos de soja pelo tratamento com fungicidas e efeitos na emergência e no rendimento de grãos. Summa Phytopathologica, Jaboticabal, v. 26, n. 3, p. 341-346, 2000.

11. LACERDA, A. L. C. et al. Armazenamento de sementes de soja dessecadas e avaliação da qualidade fisiológica, bioquímica e sanitária. Revista Brasileira de Sementes, v. 25, n. 2, p. $97-$ 105, 2003.

12. MARCHI, J. L., CICERO, S. M., GOMES JUNIOR, F. G. Utilização da análise computadorizada de plântulas na avaliação do potencial fisiológico de sementes de amendoim tratadas com fungicida e inseticida. Revista Brasileira de Sementes, Brasília, v. 33, n. 4, p. 652-662, 2011.

13. MARCOS FILHO, J. Teste de envelhecimento acelerado. In: KRZYZANOWSKI, F. C.; VIEIRA, R. D.; FRANÇA NETO, J. B. (Ed.). Vigor de sementes: conceitos e testes. Londrina: ABRATES, 1999. p. $1-24$.

14. Pereira, C. E., OliveirA, J. A., eVAngelista, J. R. E., Botelho, F. J. E., OliveirA, G. E., TRENTINI, P. Desempenho de sementes de soja tratadas com fungicidas e peliculizadas durante o armazenamento. Ciência agrotecnica, Lavras, v. 31, n. 3, p. 656-665, 2007.

15. PINTO, T. L. F., CICERO, S. M., FRANÇA-NETO, J. B., DOURADO NETO, D., FORTI, V. A. Fungicidas foliares e a doença ferrugem asiática na produção e na qualidade de sementes de soja. Revista 
Brasileira de Sementes, v. 33, n. 4, p. 680-688, 2011.

16. SANTOS, J.P. Controle de pragas durante o armazenamento de milho. Sete Lagoas: EMBRAPACNPMS. 2006. 20p. (Circular técnica, 84).

17. VIEGAS, E. G., NASCIMENTO, F. G., MEYRELLES, B. G., ROSSETTO, C. A.V. Qualidade fisiológica de sementes armazenadas de amendoim influenciada pelos produtos sintéticos e de origem vegetal. Revista Brasileira de Plantas Medicinais, Botucatu, v. 7, n. 3, p. 79-85, 2005. 